\title{
A Relative Age Test of Pre-Main Sequence Evolutionary Models Based on the Young Quadruple System GG Tauri
}

\author{
Russel J. White \\ Department of Astronomy, University of Texas at Austin, R.L.M. Hall \\ 15.308, Austin, TX 78731
}

\begin{abstract}
The components of the young hierarchical quadruple GG Tau, which span a wide range in spectral type (K7 - M7.5), are used to test both evolutionary models and the temperature scale for very young, low mass stars under the assumption of coeval formation. Of the evolutionary models tested which extend into the substellar regime, those of Baraffe et al. yield the most consistent ages when combined with a temperature scale intermediate between that of dwarfs and giants. The Palla \& Stahler and Siess et al. models are also capable of yielding a coeval age down to their lowest mass $\left(0.1 \mathrm{M}_{\odot}\right)$. These latter two models, which extend to much higher masses than the Baraffe et al. models, agree reasonably well with the Baraffe et al. models at $1.0 \mathrm{M}_{\odot}$ and thus could be combined to construct a set evolutionary models that extends from substellar to several solar masses.
\end{abstract}

\section{Introduction}

The masses and ages of young, low mass stars are primarily inferred from the comparison of their observationally determined stellar temperatures and luminosities to the predictions of theoretical pre-main sequence (PMS) evolutionary models (e.g., D'Antona \& Mazzitelli 1997). Unfortunately, these mass and age estimates are currently very imprecise because of the uncertainties in the input physics of evolutionary models. Additionally, it is unclear whether a temperature scale similar to dwarfs or giants is more appropriate for these moderately over-luminous young stars. The uncertainties in both the evolutionary models and the temperature scale lead to variations by factors as large as 10 in age and 2 in mass.

The relative ages of young binary stars offer a means of assessing the validity of evolutionary models. Assuming that the components of binary stars form simultaneously, a correct evolutionary model and temperature scale should yield the same age for all components. This test of models is pursued here using the relative ages of the PMS quadruple GG Tau, a hierarchical quadruple comprised of two binary stars in Taurus-Auriga $(\mathrm{D}=140 \mathrm{pc})$. The close pair, GG Tau A, with components $\mathrm{Aa} \& \mathrm{Ab}$ (separation $0^{\prime \prime} 25$ ) is separated by $10^{\prime \prime} 1$ from a wider pair, GG Tau B, with components Ba \& Bb (separation 1".48). Spatially separated optical spectra of GG Tau Aa and Ab were obtained using FOS aboard the Hubble Space Telescope, and of GG Tau Ba and Bb using LRIS and HIRES 
on the 10-m W. M. Keck telescopes. These spectra are presented in White et al. (WGRS) are are used in conjunction with high spatial resolution photometry (Ghez, White \& Simon 1997) to determine the stellar properties of each component separately. These properties are then used to test 4 recently available evolutionary models, 2 of which extend down to substellar masses (D'Antona \& Mazzitelli 1997, DM; Baraffe et al. 1998, BCAH) and 2 of which extend down to $0.1 \mathrm{M}_{\odot}$ (Palla \& Stahler 1999, PS; Siess et al. 2000, SDF).

\section{The Stellar Properties of GG Tau}

The angularly resolved spectra of all four components confirm that they are $\mathrm{T}$ Tauri stars and very likely to be physically associated (see WGRS). The spectral types are inferred via comparison with dwarf and giant spectra (WGRS; Luhman 1999). Gravity sensitive features in the spectra suggest surface gravities intermediate between that of dwarfs and giants. The line-of-sight extinctions and stellar luminosities are rederived following the methodology outlined in WGRS (Table 1). The masses are inferred from the BCAH models adopting dwarf temperatures for GG Tau $\mathrm{Aa}$ and $\mathrm{Ab}(4000$ and $3770 \mathrm{~K})$, and temperatures which make GG Tau $\mathrm{Ba}$ and $\mathrm{Bb}$ coeval at $1.5 \mathrm{Myr}(3020$ and $2815 \mathrm{~K})$. GG Tau Bb, with a mass of $0.043 \pm 0.005 \mathrm{M}_{\odot}$, is the lowest mass, spectroscopically confirmed T Tauri star in Taurus-Auriga; it is a T Tauri brown dwarf.

Table 1. The Stellar Properties of GG Tauri

\begin{tabular}{llccc}
\hline \hline GG Tau & $\begin{array}{l}\text { Spectral } \\
\text { Type }\end{array}$ & $\begin{array}{c}\mathrm{A}_{V} \\
(\mathrm{mag})\end{array}$ & $\begin{array}{c}\log (L) \\
\left(L_{\odot}\right)\end{array}$ & $\begin{array}{c}\text { BCAH Mass } \\
\left(M_{\odot}\right)\end{array}$ \\
\hline $\mathrm{Aa}$ & $\mathrm{K} 7 \pm 1$ & $0.72 \pm 0.26$ & $-0.074 \pm 0.067$ & $0.78 \pm 0.10$ \\
$\mathrm{Ab}$ & $\mathrm{M} 0.5 \pm 0.5$ & $3.20 \pm 0.18$ & $-0.146 \pm 0.051$ & $0.68 \pm 0.03$ \\
$\mathrm{Ba}$ & $\mathrm{M} 5.5 \pm 0.5$ & $0.00 \pm 0.26$ & $-1.196 \pm 0.104$ & $0.11 \pm 0.02$ \\
$\mathrm{Bb}$ & $\mathrm{M} 7.5 \pm 0.5$ & $0.00 \pm 0.24$ & $-1.832 \pm 0.104$ & $0.043 \pm 0.005$ \\
\hline \hline
\end{tabular}

\section{A Test of PMS Evolutionary Models}

In order to place the components of GG Tau onto an H-R diagram for comparison with evolutionary models, the observed spectral types need to be converted into effective temperatures. Unfortunately, the spectral type - effective temperature relation for $\mathrm{T}$ Tauri stars is not well known. Since their surface gravity appears to be intermediate between that of dwarfs and giants, the correct $\mathrm{T}$ Tauri temperature scale is likely to be constrained between that of dwarfs and giants. In Figure 1, the components of the GG Tau system are plotted on several H-R diagrams using both dwarf (Leggett et al. 1996) and giant (Perrin et al. 1998) temperature scales. A dotted line connects the dwarf and giant temperatures, identifying the range of plausible temperatures for each component. As the Figure illustrates, the dwarf and giant temperature scales are essentially 
identical for spectral types near $\mathrm{M} 0$, but diverge significantly for cooler spectral types. GG Tau Aa and Ab are coeval according to all evolutionary models and either temperature scale. The isochrone defined by their average age (long dashed line in Figure 1) can therefore be used to to test the evolutionary models at cooler temperatures.

The DM models predict an age for GG Tau Ba that is inconsistent with its hotter companions. It is coeval with $\mathrm{Aa}$ and $\mathrm{Ab}$ only at a temperature $\sim 200$ $\mathrm{K}$ below the dwarf temperature. The BCAH models are more successful. They predict coeval ages for both $\mathrm{Ba}$ and $\mathrm{Bb}$ using a temperature scale that is hotter than the dwarf scale by $95 \mathrm{~K}$ at M5.5, and by $225 \mathrm{~K}$ at M7.5. Although the PS and SDF models do not extend to the low dwarf temperatures of GG Tau $\mathrm{Ba}$, a small extrapolation of these models suggests that $\mathrm{Ba}$ would be coeval with $\mathrm{Aa}$ and $\mathrm{Ab}$ using a dwarf temperature for the SDF models and a temperature slightly hotter than the dwarf scale for the PS models.

These results favor the use of the BCAH models for calculating the masses and ages of the lowest mass young stars and brown dwarfs. These models should be used with an 'intermediate' temperature scale for M spectral types such as the one proposed by Luhman (1999). Although the PS and SDF models are capable of yielding consistent ages down to their lowest mass track $\left(0.1 \mathrm{M}_{\odot}\right)$, these models incorporate a grey atmosphere that, as admitted by the authors, is less realistic for masses below $\sim 0.4 \mathrm{M}_{\odot}$. At higher masses, there is no clear preference for one model over the others. All three predict masses that are consistent with dynamically inferred values (Simon, Dutrey, \& Guilloteau 2000 ). It is often necessary to estimate masses and ages for a sample of stars whose masses span the range from substellar to several solar masses. For such an application, the BCAH models could be used up to masses of $1.0 \mathrm{M}_{\odot}$ and either the PS or SDF at larger masses. Both the PS and SDF models agree reasonably well with the $\mathrm{BCAH}$ models at $1.0 \mathrm{M}_{\odot}$ (Figure 1).

I am grateful to N. Ried, G. Shultz, and especially A. Ghez for help in carrying out this project, and to M. Simon for a critical reading of this article.

\section{References}

Baraffe, I., Chabrier, G., Allard, F., \& Hauschildt, P. H. 1998, A\&A, 337, 403

D'Antona, F. \& Mazzitelli, I. 1997, Evolution of Low Mass Stars in Cool Stars in Clusters and Associations, ed. R. Pallavicini \& G. Micela, Mem. S. A. It., 68,807

Ghez, A. M., White, R. J., \& Simon, M. 1997, ApJ, 490, 353

Leggett, S. K., Allard, F., Berriman, G., Dahn, C. C., \& Haushildt, P. H. 1996, ApJS, 104, 117

Luhman, K. 1999, ApJ, 525, 466

Palla, F., \& Stahler, S. W. 1999, ApJ, 525, 772

Perrin, G. et al. 1998, A\&A, 331, 619

Siess, L., Dufour, E., \& Forestini, M. 2000, A\&A, in press

Simon, M., Dutry, A., \& Guilloteau, S. 2000, A\&A, accepted

White, R. J., Ghez, A. M., Reid, I. N., \& Schultz, G. 1999, ApJ, 520, 811 


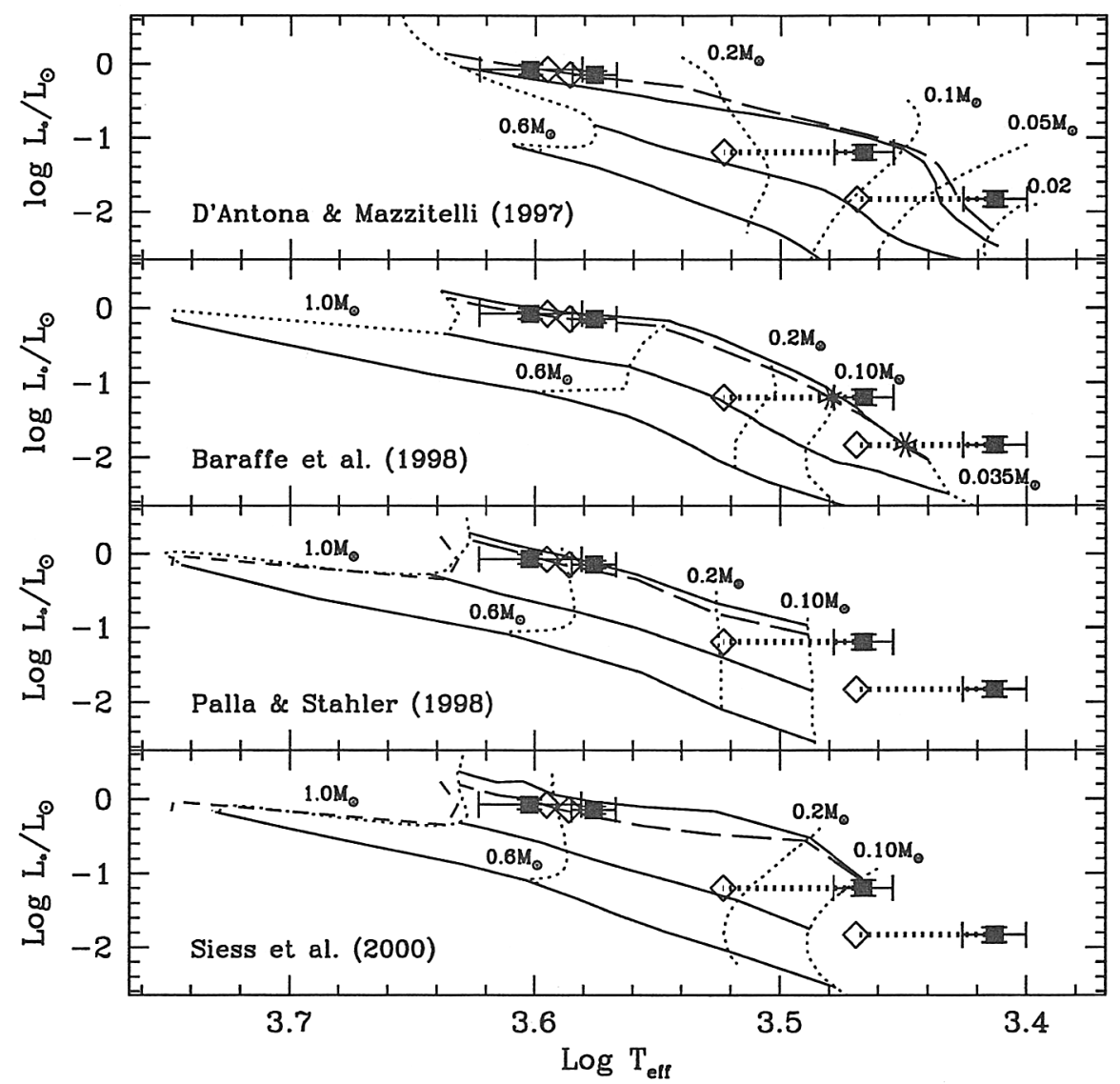

Figure 1. The locations of the GG Tau components are shown on an HR diagram using a dwarf temperatures scale (solid squares; Leggett et al. 1996) and a giant temperature scale (open diamonds; Perrin et al. 1998). Also plotted are current evolutionary models (see text) showing isochrones at $10^{6}, 10^{7}$, and $10^{8}$ yrs. The mean age of the two hottest components define an isochrone (long dashed line) which can be used to test the models at lower masses. The Baraffe et al. models provide the most consistent ages using a temperature scale intermediate between that of dwarfs and giants (asterisks). The $1.0 \mathrm{M}_{\odot}$ mass track of Baraffe et al. is over-plotted on the Palla \& Stahler and Siess et al. models to show the reasonably good agreement. 